\title{
Ethical Implications and Challenges in using Social Media: A Comprehensive Study
}

\author{
Mohammad M. Qabajeh* \\ Department of Computer Science, Faculty of Applied Science, Palestine Technical University Kadoorie, 1524200, Palestine
}

\begin{tabular}{l} 
A R T I C L E I N F O \\
\hline Article history: \\
Received: 05 November, 2021 \\
Accepted: 12 January, 2022 \\
Online: 25 January, 2022 \\
\hline Keywords: \\
Social media \\
Ethics \\
Privacy \\
Society \\
Digitization \\
\hline
\end{tabular}

\begin{abstract}
A B S T R A C T
The technological revolution penetrates every aspect of our dailylives; it changed our lives in different fields such as communication, decision-making, information access and work environment. These changes have benefits, but they also have costs. These costs include many ethical and social problems that need more investigation. The human element is the most affected part of this technological revolution; it affects our sense of privacy, our concepts of ownership, our consumption patterns and human relationships. A comprehensive review of various ethical concerns of using social media and how they affect our lives has been outlined in this paper. This study gives some recommendations and advices that help the society and reduce the heinous crimes that take place over social media.
\end{abstract}

\section{Introduction}

Recently, the emerging technology breaks through in different fields including Internet of Things (IoT), robotics, artificial intelligence, block chain, big data, cloud computing, autonomous vehicles, 3-D printing, nanotechnology, biotechnology and quantum computing. Mobile technology has grown rapidly over the past few years and the capacities of cellular networks are developed from $2 \mathrm{G}$ to $3 \mathrm{G}, 4 \mathrm{G}$ and $5 \mathrm{G}$. This increase in network capacity is engaged with unprecedented processing power and huge storage capacity of the mobile devices. This leads to high increase on number of mobile phone users to reach 5.22 billion users in October 2020 [1], which is around 70 percent of the world population.

The Internet service gains increasing popularity nowadays by all generations as a forum for communication and self-expression in the society. The number of people using the Internet around the world has grown to 4.66 billion in October 2020 [2]. The use of Internet varies from browsing the web, downloading music and watching movies, buying and selling goods and products and playing games. The Internet is also a source for social networking, which is recently becoming increasingly popular [3].

Social media platforms such as Facebook and LinkedIn use websites and applications to communicate informally with others, find people, connecting with others through groups, developing social and professional contacts, promote business and share

*Corresponding Author: Mohammad M. Qabajeh, mohammad.qabajeh@ptuk.edu.ps similar interests. Moreover, some platforms such as Twitter and Tumblr are used to for posting very short entries or updates on a social networking site, share and create hashtags and send direct messages. Other platforms allow users to publish and share photos with others either publicly or privately such as Instagram, Snapchat, Pinterest, and Flickr [4].

Social media is easily accessible by people from all ages. In a recent study [1], the number of people around the world that use social media each month is more than 4 billion, and every day an average of nearly 2 million new users are joining these platforms. People around the world are spending more time on social media too; the typical users roughly spending 15 percent of their life using social media platforms, exploring the social networking websites and online chat rooms, downloading photos, and playing games [1], [5].

The lockdowns during COVID-19 pandemic affects the habits and behaviors of most of people in different ways. It is clear that the time spent in browsing the Internet has been significantly increased. Also, the time spent on watching movies and streaming service such as Netflix and Disney+ increased compared with the time spent on watching broadcast and cable channels, since the Netflix gets 16 million new sign-ups during COVID-19 pandemic [1], [6]. In addition, YouTube videos reach four billion video views a day and it is considered as the second largest search engine after Google. At the same time, the use of social media increased to interact with family and friends and helped in boosting moods and sense of wellbeing during the lockdowns. 
It is a truth that social media become one of the megatrends affecting our everyday life; it gives us both opportunities and challenges. However, there are also significant risks that come with it, especially the ethical issues. Organizations, governments and individuals gather information through several means including search engines, invisible data gathering mechanisms, and marketing platforms. The information that is collected from several sources can be used to build complete profile for each user. Since available information on social media are globally accessible, some individuals and organizations can use this information for several purposes such as political issues, election and marketing. Hence, this available data can be increasingly utilized by most companies in the future [7,8].

Many researches have examined the ethical issues of social media including $[1,6,7]$. These studies were not covering all the ethical issues and challenges arised recently. In addition to the recent effects of COVID-19 pandemic on using social media, which are not covered in the recent research articles.

Most people have their own ethics and morals and they have their own beliefs as to what is right and what is wrong. However, the ethical concerns of using social media is increased [9]. In this study, section 2 investigates the unethical practices on social media platforms and how they affects individual physical and mental wellbeing. We also discussed the challenges that face the users in many ways including personal, social, behavioral and ethical challenges. Section 3 provides some discussions and recommendations. In section 4 some concluding remarks are given.

\section{Literature Review}

Many published research papers dealing with the ethical issues in digitization and information technology, but they did not cover all the issues raised when the individuals use new applications and platforms. Also, they do not give recommendations and advices for the users of such platforms specially for users with poor background in information technology. This paper highlights a wide range of the unethical issues that rise with the increased usage of the social networks and digital technology. In addition, we provide technical recommendations to prevent or reduce the victims of this unethical activities in the future. Here, we will give an overview about the similar efforts from the research community that have relevance to this paper.

In [10], the social and ethical issues that arise when using technology is discussed in different fields including Internet of Things, robotics, biometrics, persuasive technology, virtual \& augmented reality, and digital platforms. The paper analized different ethical concerns including privacy, security, autonomy, justice and human dignity.

Authors in [11] discussed the ethical implications of social media. This review shows the potential risks associated with using social media by psychotherapists. They also highlight that the absence of guidance and better understanding of using social media by psychotherapists can lead to inadvertent self-disclosures to clients and can place both the client and clinician at risk.

Authors in [12] showed the influence of social media on business and the effects on the sales process. They discussed how the social media has directly affect sales and marketing and highlights the challenges of using such technology.

Different types of unethical practices on social media are studied and discussed in [3]. This paper identifies the physical and mental effects of using such platforms. These unethical activates include depression, suicidal thoughts, stress, family reputation and disagreement. The authors highlighted the role of the community, government, and family in reducing the overcoming unethical activities resulted when using social media.

In [13], a discussion about the spread of using social media among people with mental disease is presented. They consider the impact of social media on mental wellbeing, they also study the risks, possible harms, and needed safety precautions with using social media for mental health. Many of the people with mental diseaseS use social media to share their experience with mental illness, requesting support from others, and to search for information about treatment recommendations, accessing mental health services and coping with the mental illness symptoms

\section{Ethical Issues Of Social Media Platforms}

Social media is now becoming more and more popular since it facilitates the sharing of ideas, information, business interests and other forms of expression and communication via virtual communities and networks. The backbone of social media is the activities of the users through sharing contents, posting of text and comments and posting digital photos or videos. This data may be sold to organizations for marketing purposes. Using social media in unethical way resulted in a violation of individual privacy and affected both information and physical security. In this section, we will discuss some of the challenging issue that may result from the unethical use of social media.

\subsection{Privacy and Confidentiality}

The rapid development of technology changes the way that we live and work. Such technologies include global positioning system (GPS) devices, smartphones, digital cameras, databases, bigdata handling and greater Internet access. This technology can document every aspect of our lives and we do not know what people knows about us and how they can use that information. In October 2020, the number of users of Internet reach 4.66 billion and the users of social media reach 4.14 billion [1]. Unfortunately, not all of them adhere by the rules of the community and behave in ethical way.

Privacy is defined as to be free from intrusion, to be free from secret surveillance, or unwanted disclosure of personal data or information by government, organizations, or individual [9]. To protect our privacy, we have to understand first what type of information can be collected and what the risks is and problems may rise when using this data. For professional IT users, they understand the problems and they can plan to protect their privacy. While, the ordinary users do not know how to protect their privacy. The privacy threats come in several categories:

- The intentional use of our personal information from the government or from private sector (in the government sector primarily for law enforcement and tax collection, and in the private sector primarily for marketing and decision making). 
- Unauthorized use or release by "insiders," the people who maintain the information.

- Theft of information.

- Inadvertent leakage of information through negligence or carelessness.

- Our own actions (sometimes intentional trade-offs and sometimes when we are unaware of the risks).

To protect our privacy from unethical and crazy social media and Internet users, we have to protect things that we believe that they should be prevented from being accessed online and not being shared with others. Some private information includes our financial data, our photos and personal information, addressing information, family issues, religious thoughts and beliefs, our political views, and our travel plans.

\subsection{Cyberbullying}

Cyberbullying means using technology to harass, threatening, torment or humiliation by a person or group upon another person (the victim) via using Internet or cell phone [10]. The accessibility and the amount of time that people spend on the Internet and social media has increased significantly. This led to new opportunities for online aggression due to several reasons including problematic use, talk to strangers, competition for status and esteem, dealing with fake social media accounts, sexting by sending sexually suggestive or explicit text or photos to boyfriends or girlfriends. Based on [3, 11, 12], Facebook and Twitter are the two highest platforms for cyberbullying.

There are several forms of cyberbullying, including and not limited to the following:

- Impersonate the victim by sending messages to threaten him and sending inappropriate messages

- Stealing the password of the victim and updating his profile to include inappropriate material such as sexual, racist, homophobic, or posting inappropriate material that attracts the attention of undesirable people.

- Using the social media pages of the victims to post personal or false information related to the victim.

- Creating social networking account or building a web site to threaten or humiliate the victim.

- Taking personal photos or inappropriate videos of the victim and posting them online or sending them via cell phone.

- Using interactive games to send unethical messages while playing and pass these messages between the players.

There are different forms of cyberbullying, which makes it difficult to identify. This leads to a situation that children's, youngsters and females be the most targeted victims of cyberbullying. Unfortunately, few of them tell their parents and relatives when they became victims of cyberbullying. All the victims should be educated and be aware of the potential effects of cyberbullying in order to prevent their privacy violation and stop cyberbullying from happening and worsening. In addition, they need to know the consequences of their actions during using social media .
Cyberbullying has direct harmful consequences on health such as emotional distress, mental and behavioral health issues, embarrassment, isolation from other community members, anxiety, and depression and in some cases it can lead to an increased risk of suicide.

\subsection{Anonymity}

Nowadays, social networking supports exchange of news, ideas, opinions, discussions, rumors, and other information with large number of followers and users. However, users of such networks must often take ethical decisions about the efficient use of this amazing freedom and power.

Anonymity is defined as the expression of opinions and ideas by persons who do not show their identity [3]. In any democratic society, the freedom of speech and expressing your opinion without fear of punishment and reprisal is essential right. However, communication without showing the identity may be exploit to commit a crime or illegal and unethical activities. Hence, it is unethical for someone not to present himself or represent himself with wrong affiliations, credentials or expertise, it is unethical to become anonymous ad showing himself to be someone different way .

In cyberspace, users use anonymity and confidentiality for harassment, extortion and fraud, to distribute pornography for children's, to threaten victims, to steal business documents or other personal information, and to infringe copyrights. Anonymity can seriously damage personal relations, damage business and incite violence. In addition, anonymity makes it difficult to track wrongdoers.

\subsection{Defamation}

Recently, the rapid increase of social networks and content gathering websites increased the risk of slanderous and reaching of false statements to broad audience. Defamation involves writing or saying something about someone, group of people, or small company that injures or damages their reputation. Defamation comes in the form of libel, or written defamation, and slander, or verbal defamation. Our Facebook status update or tweet may receive dozens of 'likes', comments and views by tens of thousands of users. So, it's important that the potential victims, users and sharers fully understand the law of defamation and impact of online defamation [13].

It's very easy for users to re-publish posts by sharing them and re-tweeting materials in a short period of time and re-broadcast it over social media. The users may fall in the trap when using the social media, they feel that they are free to express anything they want and after that find themselves liable for defamatory publications. Therefore, it is easy that a user may become a defendant and find himself faced with claims of defamation because of this posts or comments that have different meanings in different cultures for different people. Therefore, when users creates, reposts, shares, or forwards defamatory content, they have to know that they will be strictly liable for these publications. Therefore, they need be careful and review the content they are publishing since it may contain defamatory imputations concerning the aggrieved [14]. 


\subsection{Cyberstalking}

Nowadays, many social media users freely share their feelings and desires, post personal information, share location information and publish family photos. However, if they are not aware of the danger of social networking, this can lead to numerous undesirable consequences such as cyberstalking. Cyberstalking is defined as harassment or stalking that takes place over social media or the Internet in general. The victims might be individuals, groups, or even organizations and can take different forms including threats, frighten, defamation and slander. The motivation behind this action may be to control or threaten the victim or to gather information that may be used in other crimes such as identity theft or offline stalking. This kind of crimes are naturally planned and continued over a period of time [15].

When you feel that you are a victim of cyberstalking, it is important to gather as much evidence as possible and immediately make a report and document the harassment as it goes on. Also, you should block any person who you wish to stop hearing from and inform the involved platform about their abusive behavior to technically protect you from cyberstalking. Additionally, you should contact the local authorities if you feel that you need protection.

\subsection{Violence and Extortion}

Violence on the social media platforms is a widespread and continuous issue due to the large number of users of it and the divergence of the user's ideas and orientations because there is a wrong understanding of the freedom that exists in the Internet space. So, we find some users resorting to pseudonyms and concealing their personalities, and it is no longer surprising that we read insulting and insulting expressions in the dirtiest terms, whether by users' peers or celebrities, direct threats, or publishing offensive images using photo and video editing programs.

Electronic violence is defined as violence against the other, whether through electronic extortion, photography, verbal threat, or writing, and it targets all groups, especially women, whether through photography or recording conversations, messages, or offensive clips. The forms of electronic violence specially against women comes in several forms. In cases of extortion, the Snapchat application is considered a window for women's freedom and a door to violence after the emergence of some applications that provide downloading video clips and images from this application, which made it easier for the extortionist to extort women because of the clips they publish and a video of her without knowing who the perpetrator is when he threatens her or publishes her in case he does not agree to his requests.

\subsection{Sexual Harassment}

Sexual Harassment is the use of social media sites to send sexual material that harassment the recipient, this material may be messages to identify the recipient for sexual purposes, messages that contain sexual expressions, sharing of lewd jokes, sending pictures or video scenes, or sending threats and blackmail using pictures, or using pictures without the consent of the owner or without his knowledge, and sharing it through the means of communication .

There are various factors and motives behind the phenomenon of electronic harassment. These factors include the reducing of family control, looking at a woman as a body, ease of anonymity, desire for revenge with an inability to cope, sexual deprivation, incorrect childhood and lack of awareness, culture shock and constant irritation across the media.

Many of the victims of sexual harassment are cut off from their surroundings and escape from the society. The major complication of the phenomenon is the psychological effects that may extend for years. In addition, the victims may become more likely than others to suffer from anxiety, depression, phobias and panic.

\section{Summary and Discussion}

All technologies have important benefits and face several challenges. These challenges make using them unpleasant especially using social media. Several challenges seems to be trivial but they may have a serious effect on the mental and physical capabilities of the users. From the discussions presented in the previous sections, many points are concluded. These points are summarized as follows:

- The social media platforms including Twitter, Facebook, YouTube, Google, Instagram and Pinterest are not free tools and treat their users as products. They are designed to collect and utilize our activities an sell to others. Therefore, it is important to remember that if you do not pay for a product, you will be the product.

- Educating users specially users with limited background about information technology and the risks, dangers and threats of posting of sensitive information on social networks, and teaching them how to protect their privacy and that they have not to share their sensitive information such as contact number or detailed address, because this data can be dangerous and can harm them.

- With the increased storage media devices and intelligent databases, evert details of our lives are recorded and can last forever. Moreover, the websites and search engines record everything we visit and search. They can sell it to anyone who pay for them, and so users must protect their data especially adult users.

- Relatively few people do not carry CellPhones. Most people use CellPhones rather than laptops and PCs and they are mostly connected to the Internet. Billons of applications are downloaded to these devices without previous knowledge about the purpose and identity of the developers of such applications. These Apps can access more information than we can imagine.

- The variety of anticipated applications with the availability of tools such as sensors, call followers, motion detection, tiny cameras, data detectors and invisible microphones they can access and generate valuable information about everything 
about us. This data can craved by politicians, advertisers, companies, organizations and by individuals.

- Companies such as Apple and Google have tools called "kill switches" which can remotely enable and disable any application on our CellPhones and can delete all our data from our devices. Can you imagine what will happen if any outsider can access to this "kill switches" and operate them on our devices?

- Using social media for long time will reduce the time spent on doing physical activities (sport, reading books), change our habits and culture (respect for rules/ beliefs), and change our behavioral and mental activities (life management, interaction/ communication). Based on recent reports, the time per day spent using Internet exceeds 9 hours in some countries (Philippines, South Africa, Brazil, Colombia, Thailand), which is a troubling number. While in Japan they only connect for an average of 4 hours and 22 minutes per day.

- During COVID-19 pandemic and lockdowns, more and more people join the Internet and spent more time on social media. This will generate a new wave of digital innovations and resulting in new devices, platforms and services.

- The increased number of platforms such as Facebook, TikTok, Twitter, Snapchat, Youtupe, Instagram, WhatsApp and a lot other platforms make it difficult to secure the accounts and keep our information safe. The platforms is changing faster than we know how to understand them.

\section{Recommendations}

To minimize the challenges related to using social media some advices and recommendations can be taken.

- Using the technological approaches for protection user accounts such as encryption and filters to protect them from exploring sites that have objectionable material or pornography.

- Relationships with others on the Internet may be developed based on mutual interests without caring or knowing information about age, gender, nationality or race. Some of those others might not be a human at all. There are Artificial Intelligence programs that can simulate a human being in social media.

- Information on social media might be inaccurate and misleading, so do not take your decisions on what you read or hear on social media.

- The governments must put strict laws, heavy penalties and fines on people who misuse the social media and cyberspace. This action will reduce the heinous crimes that take place over the social media.

- The civil society organizations and governments must develop and build systematic culture to save the youth and the upcoming generations from the disorders and maladies of social media platforms by promoting cultural education.

- We have to form a balance view of the value and impact of using technology that can be difficult. Some of the values are obvious. Some of the impacts might be dramatic and frightening on people lives.

- Educating users about the ethical and legal issues related to using social media. Grasping these issues may minimize or prevent the negative incidents associated to social media. Users need to know that they are responsible for everything they are linked to and they have to think twice before they act on social media.

- Encourage users to use the social media in ethical way, treat others as you would like to be treated.

\section{Conclusion}

Widespread of using social media make it essential part of human's life everywhere. Younger generations and older generations are quickly becoming members of this technology. It enable its users to obtain and share information all over the world in a very short time. Unfortunately, the consequences of unethical use of social media are dangerous and harmful. There are many ethical issues in using social media that must be taken into deep consideration. The main objective of this paper is to determine what is the ethical issues arise in using social media platforms and how can we minimize the effect of the unethical use social media.

Most population of social media are unaware and not educated of the procedures that are needed to minimize the level of vulnerability of their sensitive data. Therefore, they should be educated about the risk of participating in social media and to use them in a responsible way. Also, the governments should have ethical and moral principles that can be applied for all users and lead to pleasant relationships regardless of nationality, race, culture, religious and political persuasion and social status.

\section{Conflict of Interest}

The authors declare no conflict of interest.

\section{Acknowledgements}

The author wish to thank Palestine Technical University Kadoorie, Palestine for their cooperation and support to publish this research.

\section{References}

[1] S. KEMP, "Global Digital Overview, Digital October," Singapore 2020, Available:

https://datareportal.com/reports/digital-2020-october-global-statshot.

[2] W. E. Forum, "Fourth Industrial Revolution , Strategic Intelligence," World Economic Forum,

https://intelligence.weforum.org/topics/a1Gb0000001RIhBEAW?tab=public ations.

[3] K. Chandra, S. Gounder, R Verma, S. S. Mudliar, "The Unethical Practices on Social Media," IOSR Journal Of Humanities And Social Science (IOSRJHSS), 22(7), 46-54, 2017, doi: 10.9790/0837-2207064654.

[4] C. Véliz, "Privacy and digital ethics after the pandemic," Nature Electronics, 4(1), 10-11, 2021, doi: 10.1038/s41928-020-00536-y.

[5] J. Nicholas, S. Onie, M. E. Larsen, "Ethics and Privacy in Social Media Research for Mental Health," Current Psychiatry Reports, 22(12). 84-112, 2020, doi:10.1007/s11920-020-01205-9.

[6] Z. Thomas, "Netflix gets 16 million new sign-ups thanks to lockdown," in BBC News, 2020, https://www.bbc.com/news/business-52376022

[7] V. Kumar, P. Nanda, "Social Media to Social Media Analytics: Ethical Challenges," International Journal of Technoethics (IJT), 10(2), 57-70, 2019, doi:10.4018/IJT.2019070104. 
[8] L. Royakkers, J. Timmer, L. Kool, R. van Est, "Societal and ethical issues of digitization," 20(2), 127-142, 2018, doi: 10.1145/3437075.3437093.

[9] N. Barrett-Maitland, J. Lynch "Social Media, Ethics and the Privacy Paradox," in Security and Privacy From a Legal, Ethical, and Technical Perspective, 70(6), 2-12, 2020, doi: 10.5772/intechopen.90906.

[10] S. Baase, A Gift of Fire, New Jersey, Prentice Hall, 2012, doi: $10.1145 / 572260.572271$.

[11] J. M. Kizza, Ethical and Social Issues in the Information Age, 6 ed. Springer International Publishing, 2017, doi: 10.1007/978-1-4757-2950-4.

[12] M. Khalid;, S. Singh;, K. Singh;, J. Jeevitha, G. P. Anand, "Medicus: A Doctor Appointment Booking System,", International Journal of Computing and Technology, 5(4), 48-52, 2018.

[13] V. R. Bhargava, M. Velasquez, "Ethics of the attention economy: The problem of social media addiction," 31(3), 321-359, 2021, doi: 10.1017/beq.2020.32.

[14] C. Lewis, "Social Media - Cyber trap door to defamation," Masaryk University Journal of Law and Technology, 9(1), 65-94 2015, doi: 10.5817/MUJLT2015-1-5.

[15] M. Pietkiewicz, M. Treder, "Cyberstalking in social media - Polish view," Journal of Modern Science, 38(3) 29-40, 2018, doi: 10.13166/jms/99217. 